ACCEPTED MANUSCRIPT • OPEN ACCESS

\title{
Relevance of Longitudinal Fields of Paraxial Optical Vortices
}

To cite this article before publication: Kayn A Forbes et al $2021 \mathrm{~J}$. Opt. in press https://doi.org/10.1088/2040-8986/abff96

\section{Manuscript version: Accepted Manuscript}

Accepted Manuscript is "the version of the article accepted for publication including all changes made as a result of the peer review process, and which may also include the addition to the article by IOP Publishing of a header, an article ID, a cover sheet and/or an 'Accepted Manuscript' watermark, but excluding any other editing, typesetting or other changes made by IOP Publishing and/or its licensors"

This Accepted Manuscript is @ 2021 The Author(s). Published by IOP Publishing Ltd..

As the Version of Record of this article is going to be / has been published on a gold open access basis under a CC BY 3.0 licence, this Accepted Manuscript is available for reuse under a CC BY 3.0 licence immediately.

Everyone is permitted to use all or part of the original content in this article, provided that they adhere to all the terms of the licence https://creativecommons.org/licences/by/3.0

Although reasonable endeavours have been taken to obtain all necessary permissions from third parties to include their copyrighted content within this article, their full citation and copyright line may not be present in this Accepted Manuscript version. Before using any content from this article, please refer to the Version of Record on IOPscience once published for full citation and copyright details, as permissions may be required. All third party content is fully copyright protected and is not published on a gold open access basis under a CC BY licence, unless that is specifically stated in the figure caption in the Version of Record.

View the article online for updates and enhancements. 


\title{
Relevance of Longitudinal Fields of Paraxial Optical Vortices
}

\author{
Kayn A. Forbes*, Dale Green, Garth A. Jones \\ School of Chemistry, University of East Anglia, Norwich NR4 7TJ, United Kingdom
}

Longitudinal electromagnetic fields generally become comparable with the usually dominant transverse components in strongly focused, non-paraxial beams. For paraxial optical vortex modes it is highlighted here how their angular momentum properties produce longitudinal fields that in general must be accounted for. First-order longitudinal components of quantized Laguerre-Gaussian modes are derived and numerically studied with respect to the paraxial parameter, highlighting light-matter and spin-orbit interactions that stem from the longitudinal fields of paraxial beams in free space. New restrictions are cast on the validity of neglecting longitudinal fields for paraxial optical vortices interacting with atoms, molecules and other nanostructures.

\section{*k.forbes@uea.ac.uk}

Introduction - The idealized plane-wave solutions to the Maxwell and Helmholtz equations are more often than not utilised to provide a theoretical understanding of light-matter interactions [1]. Beyond the strictly transverse plane-wave solutions, the Helmholtz wave equation also permits 'laser beam' solutions, such as the ubiquitous Gaussian. Unlike the exact plane-wave solution, however, these are approximate beam-like solutions, and the ensuing solutions to the Maxwell equations are also only approximate. A key property of such solutions, however, is that they are not strictly transverse in free space, and the electric and magnetic fields generally have nonzero components parallel to the direction of propagation [2]: viz longitudinal fields.

In their seminal study, Lax et al [3] highlighted how the paraxial solutions to the scalar wave equation consist of a purely transverse zeroth-order field and smaller, first-order, longitudinal components whose magnitude for Gaussian-type beams depends on the paraxial factor $\left(k w_{0}\right)^{-1}$ where $k=2 \pi / \lambda$ is the wave number and $w_{0}$ the beam waist (at the focal point). Therefore, the importance of longitudinal fields for general laser modes is correlated to the degree of focusing, where strongly focused beams exhibit larger longitudinal components in their electromagnetic fields. For very strongly confined fields the legitimate separation of polarization and spatial degrees of freedom in scalar beams breaks down, and vector solutions to the wave equation that involve non-separable combinations of spatial and polarization modes are necessary [4-6]. The important role and application of longitudinal fields in vector beams has been well-established $[7,8]$.

A realisation that has led to a highly active area of modern optics is that longitudinal components to the total fields are not simply just quantitative corrections to the zeroth-order transverse fields but can exhibit highly distinct properties that influence propagation characteristics of the light or the ensuing interactions with matter. Focused circularly-polarized Gaussian beams for example can induce orbital motion of trapped particles due to the spin-to-orbital angular momentum conversion $[9,10]$. Longitudinal fields are also responsible for the spin Hall and orbital Hall phenomena in paraxial fields [1113].

Twisted light beams or optical vortices are an extremely wellstudied type of structured laser light due to their rich angular momentum properties. For paraxial vortex modes, the individual photons can exhibit a spin angular momentum (SAM) $\sigma \hbar$, where $\sigma= \pm 1$, and orbital angular momentum (OAM) $\ell \hbar$, where $\ell \in \square$, per photon. The widespread study and application of optical vortices is summarised in the following articles [14-16]. Most studies have been concerned with the angular momentum properties of non-paraxial and longitudinal fields of twisted light, such as spin-orbit interactions of light (SOI) and the transfer to particles to cause mechanical motion [17-19]. The application of twisted light in spectroscopic applications is a burgeoning area of research [16]. The potentially unique role that longitudinal fields can play in these applications has previously been highlighted for highly focused Laguerre-Gaussian (LG) beams [20,21]. A particularly important realisation has been that the transfer of optical OAM to an atom [22] can only be correctly accounted for quantitatively if longitudinal fields of the input optical vortex are accounted for [23].

Here we derive the quantum electromagnetic field mode operators for LG beams that include first-order longitudinal field components in addition to the zeroth-order transverse fields. We highlight numerically that there are two distinct factors that influence the magnitude of longitudinal fields of optical vortices; firstly the well-known fact that a larger degree of focusing increases the longitudinal fields through a paraxial parameter $\left(k w_{0}\right)^{-1}$ weighting factor; but secondly that the angular momenta of optical vortices significantly influences the importance of longitudinal fields, highlighting both the quantitative and qualitative necessity of their inclusion even for paraxial optical vortices.

Transverse and Longitudinal Fields of Laguerre-Gaussian modes The most simple solutions to the wave equation are plane-waves, which subsequently become the transverse 
electromagnetic field solution to Maxwell's equations. In the Power-Zienau-Woolley (PZW) formulation of quantum electrodynamics (QED) [24,25] the electromagnetic field operators that couple to matter are the electric displacement field $\boldsymbol{d}^{\perp}(\boldsymbol{r})$ and magnetic field $\boldsymbol{b}(\boldsymbol{r})$. The superscript $\perp$ on $\boldsymbol{d}^{\perp}(\boldsymbol{r})$ is with reference to the fact that for a neutral system $\nabla \cdot \boldsymbol{d}=0$ [26], and highlights that in the PZW formulation of QED all coulombic interactions are mediated by transverse photons [27]. The electric field displacement plane-wave mode expansion operator for circularly polarised photons is given as [27]

$$
\boldsymbol{d}_{x, y}^{\perp}(\boldsymbol{r})=\sum_{\boldsymbol{k}, \sigma} \frac{\Omega}{\sqrt{2}}\left[(\hat{\boldsymbol{x}}+i \sigma \hat{\boldsymbol{y}}) a^{(\sigma)}(\boldsymbol{k}) \mathrm{e}^{i \boldsymbol{k} \cdot \boldsymbol{r}}-H . c .\right],
$$

where $k$ is the wave vector; $\sqrt{2}^{-1}(\hat{\boldsymbol{x}}+i \sigma \hat{\boldsymbol{y}})$ is the polarisation vector where $\sigma= \pm 1$ for left- and right-handed circularlypolarised light, respectively; $a^{(\sigma)}(\boldsymbol{k})$ is the annihilation operator; $\Omega$ is the normalization constant $\Omega=i\left(\hbar c k \varepsilon_{0} / 2 V\right)^{1 / 2}$ with $V$ the quantization volume; and H.c. stands for the Hermitian conjugate. The subscript $x, y$ on $\boldsymbol{d}_{x, y}^{\perp}(\boldsymbol{r})$ refers to the fact the fields are purely transverse to the Poynting vector, which for plane-waves in free space exactly coincides with the wave vector $\boldsymbol{k}$.

Approximate beam-like solutions to the scalar Helmholtz equation which have finite transverse profiles are also possible. Solutions to the paraxial wave equation, which makes the approximation that the transverse profile changes very little in the propagation direction (usually defined as the $z$ axis) over a wavelength, form an ansatz for the Helmholtz equation when combined with a phase factor $\mathrm{e}^{i(k z-\omega t)}$. Then electromagnetic beam-like solutions to Maxwell's equations can be found by ensuring all of Maxwell's laws are satisfied. Of course, one can take solutions to the full non-paraxial wave equation, one such case would be the Bessel beam class of optical vortices [28,29]. However, the most utilised of optical vortices are the LaguerreGaussian modes, which are solutions to the paraxial equation in cylindrical coordinates. As such, longitudinal components of LG modes are generally neglected with the justification of working within the paraxial approximation. The electric displacement field mode expansion operator for LG modes in the long Rayleigh range limit $z_{R} \quad z$, where $2 z_{R}=k w_{0}^{2}$, has previously been derived [30], and is given as $\boldsymbol{d}_{x, y}^{\perp}(\boldsymbol{r})=\sum_{k, \sigma, \ell, p} \frac{\tilde{\Omega}}{\sqrt{2}}\left[(\hat{\boldsymbol{x}}+i \sigma \hat{\boldsymbol{y}}) a_{|\ell|, p}{ }^{(\sigma)}(k \hat{z}) f_{|\ell|, p}(r) \mathrm{e}^{i(k z+\ell \phi)}-H . c.\right]$, (2)

where $f_{|\ell|, p}(r)=\frac{C_{p}^{|\ell|}}{w_{0}}\left(\frac{\sqrt{2} r}{w_{0}}\right)^{|\ell|} \mathrm{e}^{-\frac{r^{2}}{w_{0}^{2}}} L_{p}^{\mid \ell}\left[\frac{2 r^{2}}{w_{0}^{2}}\right] \quad$ is $\quad$ a radial distribution function where $C_{p}^{|\ell|}$ is a constant and $L_{p}^{|\ell|}$ is the associated Laguerre polynomial, and $\tilde{\Omega}$ now includes the normalisation factor $A_{\ell, p}^{-3 / 2}$ for LG modes. In the terminology introduced by Lax et al. [3], (2) is a zeroth-order solution to Maxwell's equations, and thus completely transverse.

In the plane-wave solutions commonly used, the total field is simply $\boldsymbol{d}^{\perp}(\boldsymbol{r})=\boldsymbol{d}_{x, y}^{\perp}(\boldsymbol{r})$. However, for any beam-like solution to the Helmholtz equation, the zeroth-order (2) is only an approximation, and the total field is $\boldsymbol{d}^{\perp}(\boldsymbol{r})=\boldsymbol{d}_{x, y}^{\perp}(\boldsymbol{r})+\hat{z} d_{z}^{\perp}(r)$ . The most direct method to calculate the first 'post-paraxial' longitudinal components is using the transversality conditions of Maxwell's equations $[19,23]$. In order to generate the firstorder longitudinal terms for $\boldsymbol{d}^{\perp}(\boldsymbol{r})$ we use Gauss's Law: $\nabla \cdot \boldsymbol{d}=0$. Thus the $z$ components $d_{z}^{\perp}(r)$ of the field can be determined via

$$
\begin{aligned}
& \int \nabla^{\perp} \cdot \boldsymbol{d}_{x, y}^{\perp}(\boldsymbol{r}) \mathrm{d} z= \\
& \sum_{k, \sigma, \ell, p} \frac{\tilde{\Omega}}{\sqrt{2}}\left[\frac{i}{k}\left(\frac{\partial}{\partial x}+i \sigma \frac{\partial}{\partial y}\right) f_{|\ell|, p}(r) a_{|\ell|, p}(\sigma)(k \hat{z}) \mathrm{e}^{i(k z+\ell \phi)}-H . c .\right] .
\end{aligned}
$$

Using Cartesian to cylindrical coordinate transformations produces the following mode expansion for a circularly polarised input which now includes the additional first-order longitudinal corrections to the zeroth-order transverse field:

$$
\begin{aligned}
\boldsymbol{d}^{\perp}(\boldsymbol{r}) & =\sum_{k, \sigma, \ell, p} \frac{\tilde{\Omega}}{\sqrt{2}}\left[\left\{(\hat{\boldsymbol{x}}+i \sigma \hat{\boldsymbol{y}})+\frac{i}{k}\left(\frac{\partial}{\partial r}-\frac{\ell \sigma}{r}\right) \mathrm{e}^{i \sigma \phi} \hat{z}\right\}\right. \\
& \left.\times f_{|\ell|, p}(r) a_{\mid\{\mid, p}(\sigma)(k \hat{z}) \mathrm{e}^{i(k z+\ell \phi)}-H . c\right] .
\end{aligned}
$$

There are some interesting features of (4): For $\ell=0$, one of the longitudinal terms is zero, but the other survives, highlighting how even longitudinal fields of a circularly polarised Gaussian beam (i.e $\mathrm{LG}_{0}^{0}$ ) exhibit a vortex of charge one structure in the $z$ direction through the phase factor $\mathrm{e}^{i \sigma \phi}$. This type of SOI is well known to occur in freely-propagating non-paraxial optical vortices [31], as well as non-vortex paraxial beams [11-13]. Another SOI is evident when the incident beam does have an LG structure and accompanying OAM. For $\ell=\sigma$ the longitudinal fields form a vortex of charge 2 ; whereas for $\ell=-\sigma$ the longitudinal field distribution exhibits a Gaussian structure with maximum intensity along the beam axis. This form of parallel and anti-parallel SAM and OAM projections, respectively, has been utilised in numerous studies $[32,33]$.

For the vast majority of applications, the electric field is sufficient to describe light-matter interactions. However, magnetic interactions can become important in the correct settings, such as in chiral optics [34]. The magnetic field mode expansion for a circularly polarised field is found using a similar approach, but with the aid of $\nabla \cdot \boldsymbol{b}=0$ : 


$$
\begin{aligned}
\boldsymbol{b}(\boldsymbol{r}) & =\sum_{k, \sigma, \ell, p} \frac{\tilde{\Omega}}{\sqrt{2} \varepsilon_{0} c}\left[\left\{(\hat{\boldsymbol{y}}-i \sigma \hat{\boldsymbol{x}})+\frac{1}{k}\left(\sigma \frac{\partial}{\partial r}-\frac{\ell}{r}\right) \mathrm{e}^{i \sigma \phi} \hat{\boldsymbol{z}}\right\}\right. \\
& \left.\times f_{|\ell|, p}(r) a_{|\ell|, p}{ }^{(\sigma)}(k \hat{z}) \mathrm{e}^{i(k z+\ell \phi)}-H . c\right] .
\end{aligned}
$$

The longitudinal magnetic fields are $\pi / 2$ out of phase with the longitudinal electric fields, a property previously recognized in being able to excite chiral nanostructues with linearly-polarized optical vortices $[35,36]$. Note that it is not a necessity to use circular polarisation in the mode expansions, the method of calculating the longitudinal components can easily account for other polarisations [37].

The Paraxial Approximation for Optical Vortices The validity of the paraxial approximation has been questioned by numerous authors previously [4,38-40], and even with respect to optical vortices interacting with atoms $[23,41]$. These works were interested in specific systems or values of $k w_{0}$. We now undertake a general analysis with respect to the paraxial parameter and possible optical angular momentum configurations. To do this most explicitly and readily yield analytic results, it is best to look at LG modes where $p=0$, furthermore these are the most utilised modes in experiments.

The electric displacement field (4) takes on the following form when $p=0$ and the differentiation with respect to $r$ is carried out (remembering we are working within the long Rayleigh range limit; we have also dropped the obvious dependencies for notational clarity):

$$
\begin{aligned}
\boldsymbol{d}^{\perp}(\boldsymbol{r}) & =\sum_{k, \sigma, \ell} \frac{\tilde{\Omega}}{\sqrt{2}}\left[\left\{(\hat{\boldsymbol{x}}+i \sigma \hat{\boldsymbol{y}})+\frac{i}{k}\left(\frac{|\ell|}{r}-\frac{\ell \sigma}{r}-\frac{2 r}{w_{0}^{2}}\right) \mathrm{e}^{i \sigma \phi} \hat{\boldsymbol{z}}\right\}\right. \\
& \left.\times f_{|\ell|, 0} a \mathrm{e}^{i(k z+\ell \phi)}-H . c\right] .
\end{aligned}
$$

The magnitude of the last longitudinal term is weighted by the factor $2 r / k w_{0}^{2}=r / z_{R}$, similarly to that of a Gaussian beam. This generally small contribution (for weakly-focused beams [42]) to the longitudinal fields is independent of the angular momentum properties of the field, and is exhibited by any field mode which possesses a Gaussian factor.

However, there are an additional two longitudinal terms in (6) that depend on the angular momentum properties of the beam. First there is the contribution dependent on the factor $|\ell| / k r$ which is the absolute value of the skew angle of the Poynting vector at a given location [43]. If the light also posesses SAM, there is the additional skew-angle-like term dependent on $\ell \sigma / k r$, which thus includes the mixing of helicity and topological charge values. The skew-angle-like term signififes a SOI of light in freely-propagating circularly polarised optical vortices in free-space, a phenomenon highlighted some time ago [44] but has seemingly received relatively little attention.

The intensity distributions as a function of beam waist of a variety of LG modes where the first-order longitudinal fields have been included with the generally used zeroth-order transverse fields is given in Figure 1 and Figure 2.

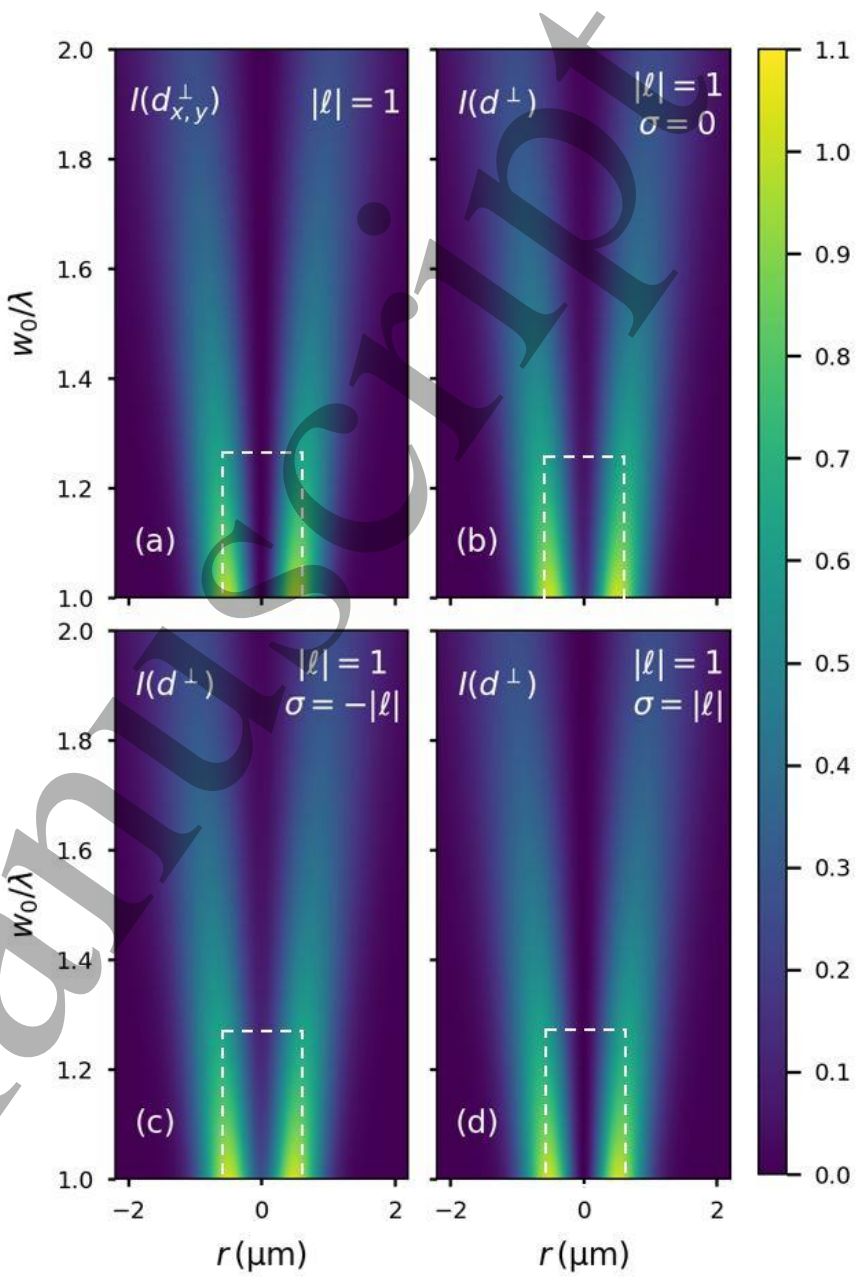

Figure 1: Intensity distributions for an LG beam with $|\ell|=1$ for a changing

beam waist (dashed boxes highlight the most prominent differences) a) the intensity contains only the transverse field components b) the total intensity including longitudinal contributions for $\sigma=0$ c) Total intensity for antiparallel OAM and SAM and d) Total intensity for parallel OAM and SAM. The range of beam waists used in Figure 1 and 2 correspond to

$$
\lambda \leq w_{0} \leq 2 \lambda \text {. }
$$

The range of beam waists used in Figure 1 and 2 correspond to $\lambda \leq w_{0} \leq 2 \lambda$, i.e. within the paraxial range $[21,38]$. Just as it is well-established that longitudinal fields cannot be neglected when unstructured (non-OAM) light is strongly focused because terms dependent on factors like $2 r / k w_{0}^{2}$ become important, Figure 1 shows that certain contributions to longitudinal fields for paraxial optical vortices cannot likewise be neglected.

The importance of these fields are still bound to specific scenarios due to the dependence on the radial distribution function, beam waist, optical angular momentum, and in comparison to the zeroth-order transverse fields are weighted by the wavelength (inverse wave number). 


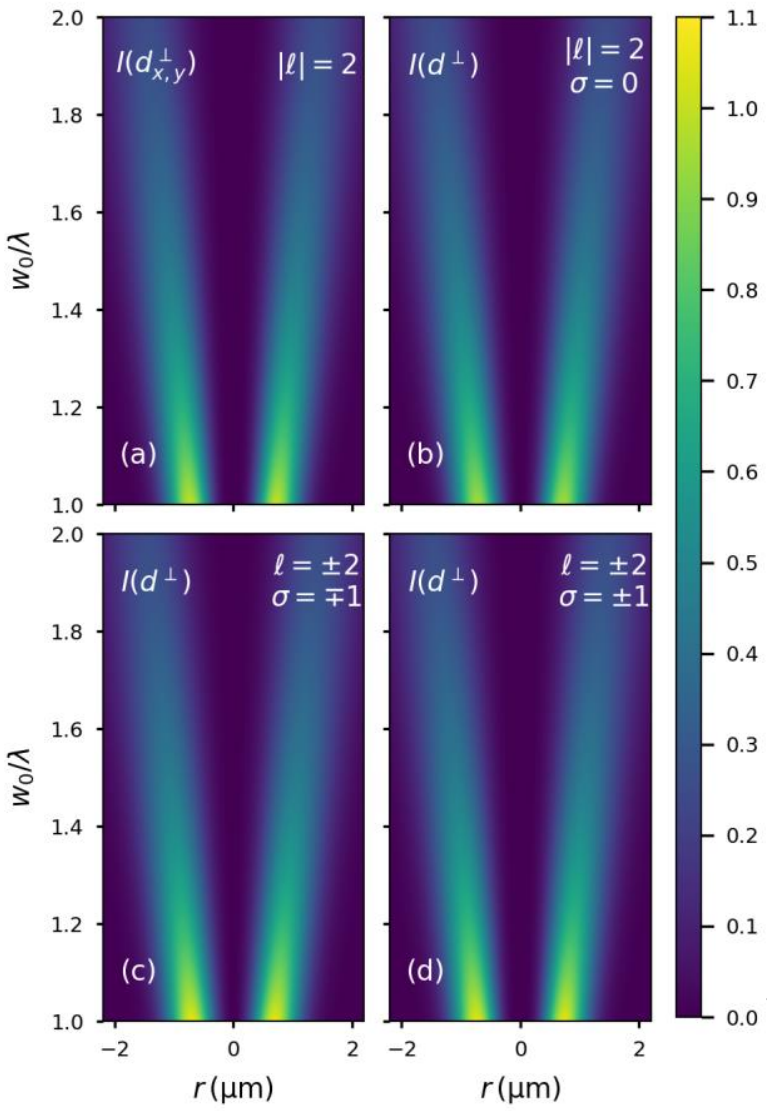

Figure 2: Intensity distributions for an LG beam with $|\ell|=2$ for a changing beam waist (a)-d) correspond to the same conditions as those in Figure 1, the range of $k w_{0}$ is also the same.

Whilst it is true that these terms do indeed become larger the more focused the beam is, Figure $1 \mathrm{~b}$ and $1 \mathrm{c}$ clearly highlights how they manifest even in LG modes for a wide range of $k w_{0}$, most clearly obvious in the so-called 'vortex core' which evidently is not truly empty for a/ range of parameters. Furthermore, if the twisted light is also circularly polarised the differences become more significant than if $\sigma=0$, and in the case of anti-parallel SAM and OAM neglecting the longitudinal fields fails for the whole range of values of $k w_{0}$ (Figure 1c). As Figure 2 shows, for higher values of $|\ell|$ the on-axis intensity distribution vanishes for paraxial beams (see Discussion), and the total field intensity distribution much more resembles the transverse only components. It is clear that the paraxial approximation (neglecting the longitudinal components of the fields) most significantly fails for $\ell= \pm 1, \sigma=0$ and $\ell= \pm 1, \sigma=\mp 1$.

Application: Single-photon absorption. The previous section shows us longitudinal fields are important to account for in spectroscopies as the highly-position-dependent intensity structure will need to be properly accounted for, particularly for high-precision measurements with optical vortices.
Single-photon absorption is the simplest optical process and is therefore an appropriate initial case to investigate the role of longitudinal fields in light-matter interactions. We may calculate the matrix element (or quantum amplitude) $M_{F I}$ of single-photon absorption in the electric dipole approximation using standard time-dependent perturbation methods, with $M_{F I}$ given by: $\left\langle F\left|H_{\text {int }}\right| I\right\rangle=\left\langle F\left|-\mathcal{E}_{0}^{-1} \boldsymbol{\mu} \cdot \boldsymbol{d}^{\perp}(\boldsymbol{r})\right| I\right\rangle$, where the initial state vector $|I\rangle=\left|n(k, \sigma, \ell, p) ; E_{0}\right\rangle$ consists of $n$ photons in the LG mode $(k, \sigma, \ell, p)$ and the material in the ground state $E_{0}$ ; the final state is given by $|F\rangle=\left|(n-1)(k, \sigma, \ell, p) ; E_{\alpha}\right\rangle$, where the mode has lost a photon and the material now exists in the excited state denoted by $\alpha ; \mu$ is the electric-dipole transition moment operator.

Using the mode expansion for the electric displacement field operator (4), the matrix element is

$$
M_{F I}=-\frac{\tilde{\Omega} \sqrt{n}}{\varepsilon_{0}}\left[e_{j}^{\mathrm{L} / R}+\frac{i}{\sqrt{2} k}\left(\frac{\partial}{\partial r}-\frac{\ell \sigma}{r}\right)\right] \hat{z}_{i} \mathrm{e}^{i \sigma \phi} f \mu_{i}^{\alpha 0} \mathrm{e}^{i(k z+\ell \phi)},
$$

where we now use suffix notation for tensor quantities and $e_{i}^{L / R}=2^{-1 / 2}(\hat{x}+i \sigma \hat{y})_{i}$ is the polarisation vector for circularly polarised light. As is standard [27], the matrix element is inserted into the Fermi rule to yield the rate $\Gamma$ of single-photon absorption as:

$$
\begin{aligned}
\Gamma & \propto\left|M_{F I}\right|^{2}=\frac{I}{2 \varepsilon_{0} c}\left[e_{i}^{\mathrm{L} / \mathrm{R}} \bar{e}_{j}^{\mathrm{L} / \mathrm{R}} f^{2}\right. \\
& +\frac{1}{k r}\left(\sigma r f f^{\prime}-\ell f^{2}\right) \hat{z}_{i}\left(\hat{y}_{j} \cos \phi-\hat{x}_{j} \sin \phi\right) \\
& \left.+\frac{\hat{z}_{i} \hat{z}_{j}}{2 k^{2} r^{2}}\left(-2 \ell \sigma r f f^{\prime}+\left(r f^{\prime}\right)^{2}+(\ell f)^{2}\right)\right] \mu_{i}^{\alpha 0} \bar{\mu}_{j}^{\alpha 0},
\end{aligned}
$$

where the intensity of the input beam is $I=n \hbar c^{2} k / A_{\ell, p}^{2} V$. The prime superscripts in (8) denote partial differentiation with respect to $r$. The first term in square brackets in (8) is the standard rate of absorption via the purely transverse zerothorder electric field, and it is therefore evident through the multitude of additional terms that accounting for longitudinal fields offer numerous additional optical interactions and qualitative corrections to the zeroth-order fields. The terms dependent on $\phi$ stem from the interferences between the transverse and longitudinal fields, and if a full beam-profile integration over $\phi$ is carried out (i.e. $\phi_{0} \rightarrow \phi_{0}+2 \pi$ ) these effects vanish. To render these observable the signals stemming from individual nanostructures or sub-domains must be resolved. The final terms are all purely longitudinal in nature, and their importance is determined by the factors discussed in the previous section. 
The rate of absorption given by (8) specifically corresponds to a nanostructure with a fixed orientation with respect to the input optical axis. It is clear that that the zeroth-order transverse fields can excite electric dipole transitions which must have allowed components in $x, y$-directions, whereas excitation through the pure longitudinal terms excite transitions which must exhibit components along the direction of propagation $z$. A similar scheme was used to map the fluorescence of molecules with specific orientations in order to precisely determine the structure of electromagnetic fields for vector vortex beams [45]. It is also worthwhile noting that individual contributions to the total rate depend on $\sigma$, i.e. the handedness of the input circular polarization; the sign of $\ell$, i.e. the handedness of the optical vortex; and the product of the two $\ell \sigma$ . Generally materials, such as molecular matter, need to be chiral in order to exhibit differential effects with respect to the sign of $\sigma$ (optical activity) through higher-order magnetic dipole and electric quadrupole interferences with the electric dipole transitions, and are thus usually weak effects [46]. The rate (8) tells us that in principle it is possible that photon absorption through purely electric-dipole transitions can yield a small differential rate for optical vortices with different helical wavefront and polarisation handedness. These differential rates that depend on the optical handedness here are comparable to standard optical activity of circularly polarised light, but in comparison to these traditional chiroptical interactions that probe the local helicity of light, the phenomena here are clearly spatial effects related to a radially varying and optical angular momentum-dependent intensity structure [47].

Orientational averaging of the particles with respect to the beam axis is done using standard methods [48], namely for a second rank tensor $\left\langle\mu_{i} \bar{\mu}_{j}\right\rangle=\frac{1}{3} \delta_{i j} \mu^{\alpha 0} \cdot \overline{\boldsymbol{\mu}}^{\alpha 0}$ :

$\langle\Gamma\rangle \propto \frac{I}{6 \varepsilon_{0} c}\left(f^{2}-\frac{\ell \sigma}{k^{2} r} f f^{\prime}+\frac{1}{2 k^{2}} f^{\prime 2}+\frac{\ell^{2}}{2 k^{2} r^{2}} f^{2}\right)\left|\mu^{\alpha 0}\right|^{2}$

The total averaged rate (9) and its individual components are plotted in Figures 3-5. It is interesting to note how an orientational average of the individual material particles also leads to the interference terms averaging to zero. Clearly the different material transition moment orientational dependences of (8) to the transverse and longitudinal fields are also lost. The SOI term dependent on $\ell \sigma$ still survives, so that even randomly oriented particles will still exhibit a small difference in the rate of absorption depending on the polarisation and wavefront handedness - this is most clear by comparing Figure $3 \mathrm{~b}$ and $\mathrm{c}$ for a given position $r$ (though note how the light must possess both SAM and OAM in this case). The average rate of absorption of an $\hat{x}$ - polarised input beam which corresponds to part (a) of Figures3-5 is calculated to be

$$
\langle\Gamma\rangle \propto \frac{I}{6 \varepsilon_{0} c}\left[f^{2}+\frac{1}{k^{2}}\left[\left(\cos ^{2} \phi\right) f^{\prime 2}+\frac{\ell^{2}}{r^{2}} f^{2}\right]\right]\left|\mu^{\alpha 0}\right|^{2}
$$

For linearly polarised light there is a longitudinal contribution to the rate of absorption dependent on $\phi$ which is spatially elongated along the direction of input polarisation. It is clear to see that the rate of absorption has an acute dependence on radial position, but also the total rate is altered by the longitudinal fields even for beams propagating within the paraxial regime. The results displayed in Figure 3 corresponds to $w_{0}=\lambda$; Figures 4 and 5 highlight the same rate but for $w_{0}=3 \lambda / 2$ and $w_{0}=2 \lambda$, respectively. It is highly relevant to note that Quinteiro et al. [23] highlighted how longitudinal fields in the specific case of parallel and anti-parallel SAM and OAM had a significant influence on atomic electric quadrupole transitions even when $w_{0} \approx 3.7 \lambda$ for an atom trapped at $r \approx 0$ (see our Figure $1 \mathrm{c}$ and $3 \mathrm{~b}, 4 \mathrm{~b}$ and $5 \mathrm{~b}$ ). Because longitudinal fields stem from the gradients of the transverse fields, it is not surprising that electric quadrupole interactions are particularly sensitive to them as they themselves are driven by electric field gradients. Clearly for such high-precision measurements dealing with trapped particles, in atomic optics in particular, longitudinal fields of optical vortices must be taken account of for a wide range of $k w_{0}$.

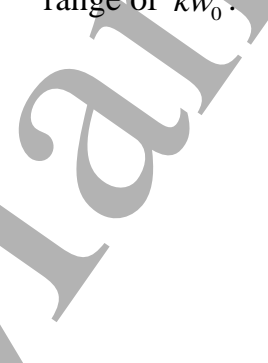



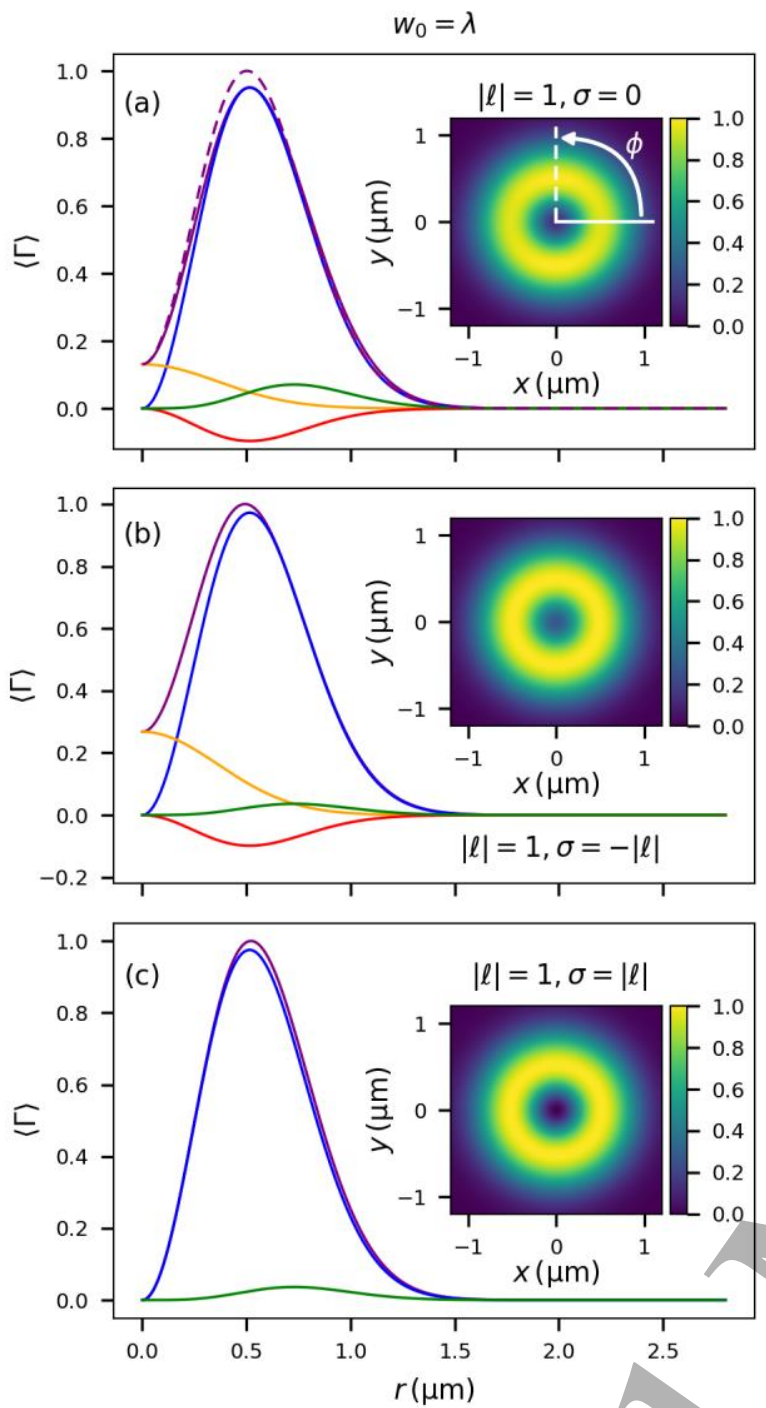

$$
\begin{array}{ll}
\text { - total }(\phi=0) & - \text { long } \propto r^{2} \\
\text { trans } \propto r^{2} & - \text { long } \propto r^{4} \\
\text { long } \propto r^{0} & -- \text { total }(\phi=\pi / 2)
\end{array}
$$

Figure 3: 1D Plots of the total and individual contributions to the rate of single-photon absorption (Eq.(9)) with 2D inset plot corresponding to total rate $\left(p=0, w_{0}=\lambda\right)$. (a) corresponds to linearly-polarised light in the $\boldsymbol{x}$ direction.
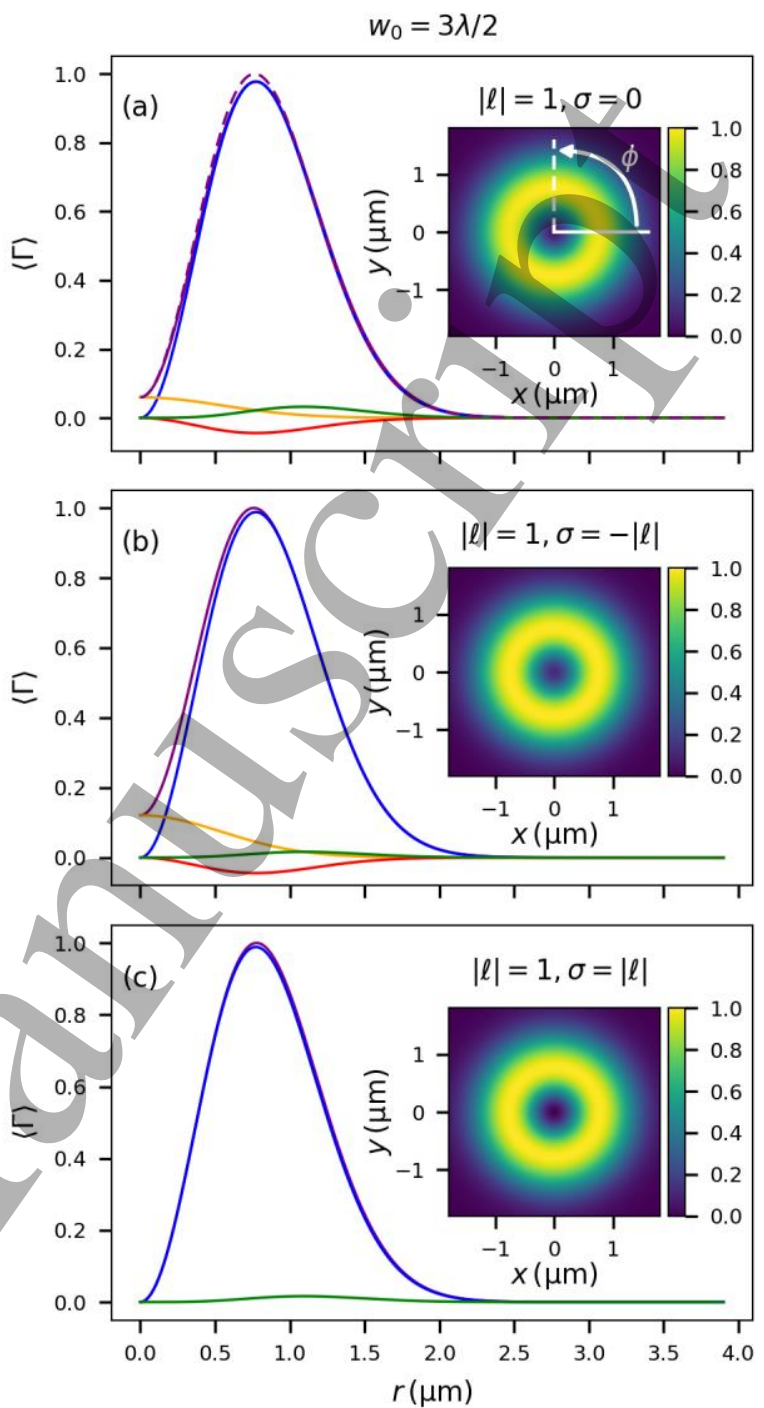

$$
\begin{array}{ll}
- \text { total }(\phi=0) & - \text { long } \propto r^{2} \\
\text { trans } \propto r^{2} & - \text { long } \propto r^{4} \\
\text { long } \propto r^{0} & ---\operatorname{total}(\phi=\pi / 2)
\end{array}
$$

Figure 4: 1D Plots of the total and individual contributions to the rate of single-photon absorption (Eq.(9)) with 2D inset plot corresponding to total rate $\left(p=0, w_{0}=3 \lambda / 2\right)$. (a) corresponds to linearly-polarised light in the $x$ direction. 

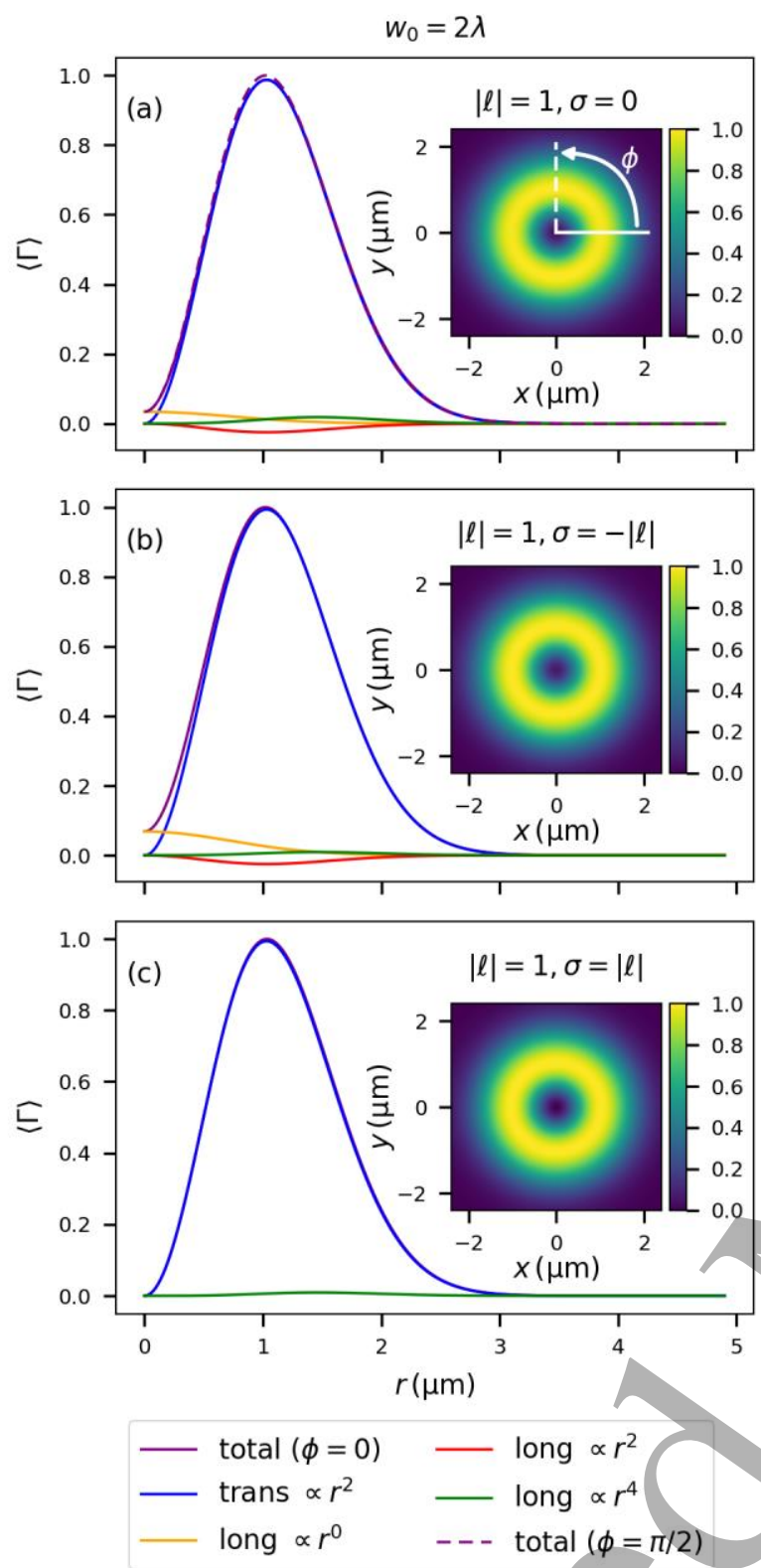

Figure 5: 1D Plots of the total and individual contributions to the rate of single-photon absorption (Eq.(9)) with 2D inset plot corresponding to total rate $\left(p=0, w_{0}=2 \lambda\right)$. (a) corresponds to linearly-polarised light in the $\boldsymbol{x}$ direction.

Discussion and Conclusion LG modes are solutions to the paraxial wave equation, and therefore must strictly be bound to any approximations associated with it. Detailed conditions of where the paraxial approximation fails for general laser modes can be found in $[38,39]$. If one wishes to rigorously account quantitatively for the longitudinal fields of very stronglyfocused $w_{0}<\lambda$ beams, then either (a) the non-paraxial solutions should be utilised [28,29], (b) a systematic expansion in the paraxial parameter in the spirit of previous studies $[3,49,50]$ or (c) explicilt inclusion of the focusing approach with high NA $[18,32,51-53]$ should be adopted. In relation to this it is worth mentioning some recent work highlighting a class of paraxial beam which exhibit large longitudinal components but under paraxial focusing conditions [54,55]. The first-order nature of the longitudinal fields this work has concentrated on means their contributions are completely valid within the paraxial regime.

It is worth discussing a subtle point about the method of deriving the electric displacement (4) and magnetic (5) fields which were derived using the divergenceless nature of electric and magnetic fields in free space. The magnetic field could have been derived using Faraday's law $\nabla \times \boldsymbol{e}^{\perp}(\boldsymbol{r})=-\partial \boldsymbol{b}(\boldsymbol{r}) / \partial t$ which yields the fields as in (5) plus second-order/corrections to the transverse field which essentially stem from $\partial^{2} / \partial z^{2}$ variations of the amplitude. Similarly the Maxwell-Ampere Law would give second-order transverse corrections to the transverse electric displacement field. Working within the paraxial approximation $\partial^{2} / \partial z^{2} \square k \partial / \partial z$ these terms have been neglected here due to the fact they only become important in situations where a paraxial description of the field would be inaccurate. It has been highlighted how for highly-focused vortex beams these second-order transverse fields exhibit unique features for $|\ell|=2$ [21,32]. Similarly, it is worth making the point that restricting our results to within the Rayleigh range is legitimate for LG modes as the $z$ dependence of the full, non-Rayleigh range LG mode function only leads to longitudinal and transverse fields which are higher-order in the paraxial parameter (i.e. second-, third-, and so on post-paraxial approximations), and thus are actually beyond the validity of the paraxial wave equation and so their inclusion is not supported here either.

The field of twisted light and optical OAM has largely been concerned with applications in mechanical nanomanipulation, communications, and quantum information studies - only in the last few years have the unique properties of twisted beams been implemented in atomic and molecular optics and spectroscopy. Here we have highlighted how in such studies, longitudinal fields of paraxial optical vortices must be accounted for in general. In particular our analysis shows that deviation from the purely zeroth-order transverse field description most appreciably occurs for optical vortex modes with $\ell= \pm 1, \sigma=\mp 1$ or $|\ell|=1, \sigma=0$ and values of $w_{0}$ around a few integer multiples of the wavelength. The most striking differences in these cases occur for nanostructures placed close to the so-called vortex singularity. Nanostructures with specific orientation with respect to the optical axis have more potential to exhibit larger and more interesting effects with the longitudinal fields than systems of randomly oriented structures. Unlike the strongly focused case $w_{0}<\lambda$ where the total longitudinal components can dominate the transverse fields, here it has been shown that for paraxial optical vortices the inclusion of first-order longitudinal fields is still important both qualitatively and quantitatively, as they introduce novel optical interactions with matter as well as alter the electromagnetic fields and corresponding position-dependent intensity structure.

K.A.F. thanks the Leverhulme Trust for funding through a Leverhulme Early Career Fellowship ECF-2019-398. 
[1] G. Grynberg, A. Aspect, and C. Fabre, Introduction to Quantum Optics: From the Semi-Classical Approach to Quantized Light (Cambridge University Press, Cambridge, U.K., 2010).

[2] A. Zangwill, Modern Electrodynamics (Cambridge University Press, Cambridge, 2013).

[3] M. Lax, W. H. Louisell, and W. B. McKnight, From Maxwell to Paraxial Wave Optics, Phys. Rev. A 11, 1365 (1975).

[4] C. G. Chen, P. T. Konkola, J. Ferrera, R. K. Heilmann, and M. L. Schattenburg, Analyses of Vector Gaussian Beam Propagation and the Validity of Paraxial and Spherical Approximations, JOSA A 19, 404 (2002).

[5] L. Novotny and B. Hecht, Principles of Nano-Optics (Cambridge University Press, Cambridge, 2012).

[6] J. Wang, F. Castellucci, and S. Franke-Arnold, Vectorial Light-Matter Interaction: Exploring Spatially Structured Complex Light Fields, AVS Quantum Sci. 2, 031702 (2020).

[7] Q. Zhan, Cylindrical Vector Beams: From Mathematical Concepts to Applications, Adv. Opt. Photonics 1, 1 (2009).

[8] B. Gu and Y. Cui, Nonparaxial and Paraxial Focusing of Azimuthal-Variant Vector Beams, Opt. Express 20, 17684 (2012).

[9] H. Adachi, S. Akahoshi, and K. Miyakawa, Orbital Motion of Spherical Microparticles Trapped in Diffraction Patterns of Circularly Polarized Light, Phys. Rev. A 75, 063409 (2007).

[10] Y. Zhao, D. Shapiro, D. McGloin, D. T. Chiu, and S. Marchesini, Direct Observation of the Transfer of Orbital Angular Momentum to Metal Particles from a Focused Circularly Polarized Gaussian Beam, Opt. Express 17, 23316 (2009).

[11] A. Bekshaev, Improved Theory for the PolarizationDependent Transverse Shift of a Paraxial Light Beam in Free Space, Ukr. J. Phys. Opt. 12, 10 (2011).

[12] A. Y. Bekshaev, Polarization-Dependent Transformation of a Paraxial Beam upon Reflection and Refraction: A Real-Space Approach, Phys. Rev. A 85, 023842 (2012).

[13] A. Y. Bekshaev, Spin-Orbit Interaction of Light and Diffraction of Polarized Beams, J. Opt. 19, 085602 (2017).

[14] H. Rubinsztein-Dunlop et al., Roadmap on Structured Light, J. Opt. 19, 013001 (2016).

[15] S. M. Barnett, M. Babiker, and M. J. Padgett, editors, Optical Orbital Angular Momentum (The Royal Society, London, 2017).

[16] M. Babiker, D. L. Andrews, and V. E. Lembessis, Atoms in Complex Twisted Light, J. Opt. 21, 013001 (2018)

[17] T. A. Nieminen, A. B. Stilgoe, N. R. Heckenberg, and H. Rubinsztein-Dunlop, Angular Momentum of a Strongly Focused Gaussian Beam, J. Opt. Pure Appl. Opt. 10, 115005 (2008).

[18] P. B. Monteiro, P. A. M. Neto, and H. M. Nussenzveig, Angular Momentum of Focused Beams: Beyond the Paraxial Approximation, Phys. Rev, A 79, 033830 (2009).

[19] K. Y. Bliokh and F. Nori, Transverse and Longitudinal Angular Momenta of Light, Phys. Rep. 592, 1 (2015).

[20] V. Klimov, D. Bloch, M. Ducloy, and J. R. R. Leite, Detecting Photons in the Dark Region of Laguerre-Gauss Beams, Opt. Express 17, 9718 (2009).

[21] V. V. Klimov, D. Bloch, M. Ducloy, and J. R. Leite, Mapping of Focused Laguerre-Gauss Beams: The Interplay between Spin and Orbital Angular Momentum and Its Dependence on Detector Characteristics, Phys. Rev. A 85, 053834 (2012).

[22] C. T. Schmiegelow, J. Schulz, H. Kaufmann, T. Ruster, U. G. Poschinger, and F. Schmidt-Kaler, Transfer of Optical Orbital Angular Momentum to a Bound Electron, Nat. Commun. 7, 12998 (2016).

[23] G. F. Quinteiro, F. Schmidt-Kaler, and C. T. Schmiegelow, Twisted-Light-Ion Interaction: The Role of Longitudinal Fields, Phys. Rev. Lett. 119, 253203 (2017).
[24] D. L. Andrews, G. A. Jones, A. Salam, and R. G. Woolley, Perspective: Quantum Hamiltonians for Optical Interactions, J. Chem. Phys. 148, 040901 (2018).

[25] D. L. Andrews, D. S. Bradshaw, K. A. Forbes, and A. Salam, Quantum Electrodynamics in Modern Optics and Photonics: Tutorial, JOSA B 37, 1153 (2020).

[26] M. Babiker, E. A. Power, and T. Thirunamachandran, On a Generalization of the Power-Zienau-Woolley Transformation in Quantum Electrodynamics and Atomic Field Equations, Proc. R. Soc. Lond. Math. Phys. Sci. 338, 235 (1974).

[27] D. P. Craig and T. Thirunamachandran, Molecular Quantum Electrodynamics: An Introduction to Radiation-Molecule Interactions (Courier Corporation, New York, 1998).

[28] S. M. Barnett and L. Allen, Orbital Angular Momentum and Nonparaxial Light Beams, Opt. Commun. 110, 670 (1994).

[29] D. L. Andrews and M. Babiker, editors, The Angular Momentum of Light (Cambridge University Press, Cambridge, 2012).

[30] L. D. Romero, D. L. Andrews, and M. Babiker, A Quantum Electrodynamics Framework for the Nonlinear Optics of Twisted Beams, J. Opt. B Quantum Semiclassical Opt. 4, S66 (2002).

[31] K. Y. Bliokh, F. J. Rodríguez-Fortuño, F. Nori, and A. V. Zayats, Spin-Orbit Interactions of Light, Nat. Photonics 9, 796 (2015).

[32] Y. Iketaki, T. Watanabe, N. Bokor, and M. Fujii, Investigation of the Center Intensity of First-and Second-Order LaguerreGaussian Beams with Linear and Circular Polarization, Opt. Lett. 32, 2357 (2007).

[33] Y. Zhao, J. S. Edgar, G. D. Jeffries, D. McGloin, and D. T. Chiu, Spin-to-Orbital Angular Momentum Conversion in a Strongly Focused Optical Beam, Phys. Rev. Lett. 99, 073901 (2007).

D. L. Andrews, Quantum Formulation for Nanoscale Optical and Material Chirality: Symmetry Issues, Space and Time Parity, and Observables, J. Opt. 20, 033003 (2018).

[35] C. Rosales-Guzmán, K. Volke-Sepulveda, and J. P. Torres, Light with Enhanced Optical Chirality, Opt. Lett. 37, 3486 (2012).

[36] P. Woźniak, I. D. Leon, K. Höflich, G. Leuchs, and P. Banzer, Interaction of Light Carrying Orbital Angular Momentum with a Chiral Dipolar Scatterer, Optica 6, 961 (2019).

[37] O. V. Angelsky, A. Y. Bekshaev, S. G. Hanson, C. Y. Zenkova, I. I. Mokhun, and Z. Jun, Structured Light: Ideas and Concepts, Front. Phys. 8, (2020).

[38] S. Nemoto, Nonparaxial Gaussian Beams, Appl. Opt. 29, 1940 (1990).

[39] P. Vaveliuk, B. Ruiz, and A. Lencina, Limits of the Paraxial Approximation in Laser Beams, Opt. Lett. 32, 927 (2007).

[40] A. Y. Bekshaev and V. M. Grimblatov, Violation of Transversality and Transfer of Electromagnetic Field Energy in Coherent Light Beams, Opt. Spectrosc. 66, 127 (1989).

[41] G. F. Quinteiro, C. T. Schmiegelow, and F. Schmidt-Kaler, The Paraxial Approximation Fails to Describe the Interaction of Atoms with General Vortex Light Fields, ArXiv Prepr. ArXiv200400040 (2020).

[42] A. Carnicer, I. Juvells, D. Maluenda, R. Martínez-Herrero, and P. M. Mejías, On the Longitudinal Component of Paraxial Fields, Eur. J. Phys. 33, 1235 (2012).

[43] J. Leach, S. Keen, M. J. Padgett, C. Saunter, and G. D. Love, Direct Measurement of the Skew Angle of the Poynting Vector in a Helically Phased Beam, Opt. Express 14, 11919 (2006).

[44] L. Allen, V. E. Lembessis, and M. Babiker, Spin-Orbit Coupling in Free-Space Laguerre-Gaussian Light Beams, Phys. Rev. A 53, R2937 (1996).

[45] L. Novotny, M. R. Beversluis, K. S. Youngworth, and T. G. Brown, Longitudinal Field Modes Probed by Single Molecules, Phys. Rev. Lett. 86, 5251 (2001). 
[46] L. D. Barron, Molecular Light Scattering and Optical Activity (Cambridge University Press, Cambridge, 2009).

[47] K. A. Forbes and D. L. Andrews, Orbital Angular Momentum of Twisted Light: Chirality and Optical Activity, J. Phys. Photonics 3, 022007 (2021).

[48] D. L. Andrews and T. Thirunamachandran, On ThreeDimensional Rotational Averages, J. Chem. Phys. 67, 5026 (1977).

[49] G. P. Agrawal and D. N. Pattanayak, Gaussian Beam Propagation beyond the Paraxial Approximation, JOSA 69, 575 (1979).

[50] M. Couture and P.-A. Belanger, From Gaussian Beam to Complex-Source-Point Spherical Wave, Phys. Rev. A 24, 355 (1981).

[51] N. Bokor, Y. Iketaki, T. Watanabe, and M. Fujii, Investigation of Polarization Effects for High-Numerical-Aperture FirstOrder Laguerre-Gaussian Beams by 2D Scanning with a Single Fluorescent Microbead, Opt. Express 13, 10440 (2005).

[52] K. Y. Bliokh, E. A. Ostrovskaya, M. A. Alonso, O. G. Rodríguez-Herrera, D. Lara, and C. Dainty, Spin-to-Orbital Angular Momentum Conversion in Focusing, Scattering, and Imaging Systems, Opt. Express 19, 26132 (2011).

[53] C. Prajapati, Study of Electric Field Vector, Angular Momentum Conservation and Poynting Vector of Nonparaxial Beams, J. Opt. (2021).

[54] A. Aiello, M. Paúr, B. Stoklasa, Z. Hradil, J. Řeháček, and L. L. Sánchez-Soto, Observation of Concentrating Paraxial Beams, OSA Contin. 3, 2387 (2020).

[55] M. A. Porras, Exploding Paraxial Beams, Vortex Beams, and Cylindrical Beams of Light with Finite Power in Linear Media, and Their Enhanced Longitudinal Field, Phys. Rev. A 103, 033506 (2021). 